«Une chevalerie sportive » mise au service de la responsabilité sociale : généalogie du projet politique de Pierre de Coubertin

Shirine Sabéran

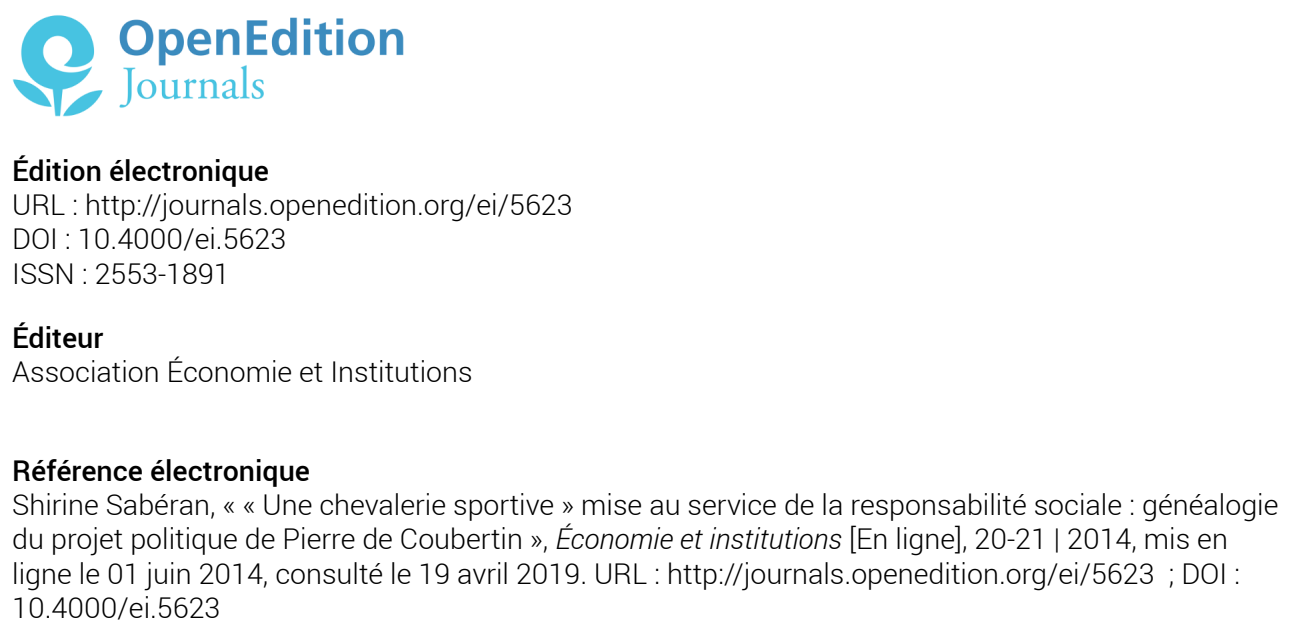

Ce document a été généré automatiquement le 19 avril 2019

Revue Économie et institutions 


\title{
«Une chevalerie sportive » mise au service de la responsabilité sociale : généalogie du projet politique de Pierre de Coubertin
}

\author{
Shirine Sabéran
}

\section{NOTE DE L'ÉDITEUR}

L'article de Shirine Sabéran (1971-2013) avait été soumis à Économie et Institutions bien avant son décès. Ce texte a suivi le parcours normal d'un article présenté à une revue académique en vue d'y être publié, si ce n'est que la dernière phase du processus de référé s'est déroulée en son absence : ce sont ses collègues et amis qui ont apporté à son texte les modifications marginales demandées par les rapporteurs ; c'est sa famille qui en a autorisé la publication.

1 Les années quatre-vingt marquent le début de la mutation du discours d'entreprise en France. L'émergence d'un vocabulaire emprunté à la compétition sportive, l'adoption de valeurs communes destinées à forger l'adhésion des salariés (Godelier 2006) naissent à l'époque où l'éthique de l'entreprise se dessine comme une alternative à l'État providence, capable de contrôler et de réguler l'économie de marché (Salmon 2002 p. 7). Depuis les années deux mille, le phénomène ne cesse de se renforcer. Les sportifs de hautniveau dispensent des formations aux salariés des entreprises ; les prétendues valeurs des sports, du rugby ou de l'aïkido, sont désormais sollicitées ; tandis que le MEDEF invite ses membres à « jouer le jeu ». L'enchevêtrement des pratiques sportives et des pratiques des affaires s'explique, d'un point de vue sociologique, parce qu'elles se retrouvent sur le terrain de la performance (Ehrenberg 1991) et parce qu'investir dans le sport-loisir permet de restaurer l'énergie consacrée au travail (Vigarello 2002 p. 68-71). Ce constat 
porterait toutefois une contradiction, parce qu'« à l'origine » le monde de l'entreprise et le monde du sport " ont tout pour s'opposer et être enfermés dans des sphères séparées ", tant le premier serait le lieu d'expression du travail et de la réalisation de profit et le second, celui du loisir et du désintéressement (Barbusse 2002 p. 392). D'un point de vue managérial, l'évolution des «métaphores de l'entreprise communautaire » utilisées dans les discours patronaux pour modeler une culture d'entreprise confirme cette tendance: après le recours à la « famille » à la fin du dix-neuvième siècle, à la « maison » à partir des années vingt, «l'équipe sportive » fait son apparition dans les années soixante-dix pour illustrer l'activité au sein de l'entreprise (Godelier 2006 pp.13-16). Associé à la performance et à l'efficacité, le sport est d'autant plus utilisé par l'entreprise qu'il est «vecteur de valeurs » et contribue à améliorer positivement la notoriété de la marque (Katz-Bénichou 2004). Plus largement, le " pouvoir symbolique » des "valeurs du sport » est mis au service des entreprises soucieuses de répondre à l'appel à la responsabilité sociale exprimé par la société civile (Bayle \& Mercier 2008).

2 La piste que nous proposons d'explorer remonte dans l'histoire des idées. Elle propose d'opérer un retour aux sources, à la fin du dix-neuvième siècle, en France, au moment où l'idée d'une responsabilité sociale des industriels, qui s'élabore dans les milieux libéraux, rencontre l'éthique sportive, et d'en retracer l'histoire intellectuelle. Cette démarche a pour objectif de discuter la thèse des «logiques convergentes" (Barbusse 2002), qui unissent sport et entreprise à cette époque, et de montrer qu'ils ne se retrouvent pas seulement sur le terrain de la performance, mais également - et surtout - sur celui de la responsabilité de la classe dirigeante. Cette démarche vise ainsi à prolonger en amont la thèse de Bourdieu qui rappelle que du monde des idées au réel, le sport moderne, dont on doit la définition à Coubertin ${ }^{1}$, est partie intégrante d'un « idéal moral » pensé à la fin du dix-neuvième siècle qui «trouve sa réalisation dans les grandes institutions d'enseignement privé » (Bourdieu 1980 p. 180). Ce retour aux sources a pour but de montrer que l'éthique sportive - i.e. l'idéal moral qui guide la pratique du sport - telle qu'elle a été pensée par Pierre de Coubertin s'est dès le départ construite sur le même terrain intellectuel que celui qui a donné naissance au paternalisme social en France (Ballet \& de Bry 2001) - terrain défriché par Alexis de Tocqueville et revisité par Frédéric Le Play. En replaçant l'éthique coubertinienne dans son corpus libéral et élitiste son rôle sera mis en évidence : accompagner la société moderne, la doter de valeurs alternatives à l'individualisme utilitaire pour construire les démocraties marchandes. Il apparaîtra ainsi que cette éthique sportive qui affiche le primat de la liberté individuelle s'enracine dans un courant réformateur qui cherche une réponse politique non étatique pour conjurer le risque de dissociation (Castel 1999 pp. 347-429) en érigeant des institutions sportives privées éducatives (Brohm 2008 pp. 45-46).

Il convient toutefois de préciser que considérer Coubertin comme un théoricien des élites (Weber 1970-71, Boulongne 1975, Clastres 2005) n'est pas sans poser problème au regard des tensions que recèle sa pensée (Ferry 1997) et des récupérations dont elle a fait l'objet (Clastres 2005). Car, en accord avec l'idéal républicain méritocratique fondé sur l'égalité des chances, l'image de la compétition sportive résonne également avec la juste concurrence marchande (Ehrenberg 1991 p. 18, Jamet 2002, Chapron 2004, Saint Martin 2004): Que tous soient égaux sur la même ligne de départ et que chacun obtienne la récompense qu'il mérite! En retenant cette devise, Ferry qualifie l'idéal de Coubertin d'«humanisme républicain» (Ferry 1997). Il oublie cependant que celle-ci s'accorde davantage avec l'idée du « sport pour tous » qui a forgé la Troisième République (Chanet 
2000), le Front Populaire (Weber 1970-71) et le compromis keyneso-fordien, qu'avec le modèle originel de Coubertin. En amont de ces deux interprétations de l'idéal de Coubertin et du rôle social accordé au sport, se profilent et s'opposent deux conceptions de la République, celle qui fait primer la liberté sur l'égalité et celle fait primer l'égalité sur la liberté. Le compromis keynéso-fordien d'après-guerre a conjugué le sport avec l'égalité ; il s'est ainsi approprié un Coubertin humaniste et pacifiste qui a fait oublier le théoricien des élites. Les écrits du pédagogue font apparaitre qu'il conjugue plus volontiers sport avec liberté et responsabilité. En cela son idéal résonne davantage avec le post-fordisme dont le renouveau éthique se construit sur une rhétorique élitiste (Salmon 2009 p. 79). «Culte du héros », « communauté d'excellence », « leadership et liberté », ces termes utilisés pour décrire le changement «d'état d'esprit » et de " paradigme » décrit par les philosophes du management (Salmon 2009 pp.79-105), s'inscrivent dans le prolongement $\mathrm{du}$ changement d'état d'esprit de la classe dirigeante française que Coubertin voulait initier par la pédagogie sportive. D'hier à aujourd'hui, le contenu éthique donné à la responsabilité de l'entreprise a bien évidemment évolué, et à ce titre, le contenu des valeurs sportives, ou supposées telles, utilisées par les discours d'entreprise également. Aussi, plutôt que de focaliser l'analyse sur le contenu des valeurs et des règles volontaires adoptées au fil des deux siècles, nous nous centrerons sur la forme des règles susceptibles de réguler la société industrielle, en tant qu'elles sont le produit de l'initiative privée des élites dirigeantes et non de l'État.

4 La pensée et l'action politique de Coubertin s'inscrivent dans cette tradition libérale française qui, tout en reconnaissant les vertus de la liberté et de l'égalité, déplore la perte des valeurs chevaleresques de la société de l'Ancien Régime et l'émergence de valeurs uniquement centrées sur la recherche de l'intérêt individuel. Héritier d'un courant qui, de Burke à Montesquieu, pose aux conservateurs progressistes le problème de la dégradation des valeurs dans les sociétés industrielles, Coubertin cherche, en lisant Tocqueville et Le Play, à le dépasser en participant à la formation d'une «chevalerie sportive » par une réforme de l'éducation inspirée des pratiques en l'Angleterre. Dans son esprit, cette chevalerie sportive fabriquée par les institutions adéquates sera à même de transmettre les bonnes valeurs et les bonnes pratiques à l'ensemble de la société. Ces bonnes pratiques sont les actions tournées vers le bien public; en cela, elles visent la réalisation d'un bien commun, au sens défini par l'Antiquité. L'identification de ce bien suit une procédure spécifique et commune chez Tocqueville, Le Play et Coubertin. Elle repose d'abord sur une méthode d'analyse fondée sur l'observation et le comparatisme, appliquée à un objet d'étude, les mœurs. Cette méthode conduit à identifier de multiples biens qui sont le produit de l'histoire de chaque peuple et adaptés au réel. Le regard particulier porté vers le monde anglo-saxon, l'Angleterre et les États-Unis, permet d'identifier ce qui a fait la réussite de ces nations et qui peut être adapté à la société française. Ces multiples biens identifiés permettent ensuite de dégager le commun parmi le multiple ${ }^{2}$. Le bien commun tocquevillo-leplaysien, dont Coubertin se fait l'héritier, est la paix sociale. Sa réalisation requiert l'instauration d'un nouveau lien social qui aurait les mêmes vertus que celui qui prévalait dans l'Ancien Régime: garantir l'unité entre les classes. Par l'édification des bonnes institutions, en particulier un système éducatif d'initiative privée, et la diffusion lente et graduelle des bonnes pratiques, ces auteurs pensent la possibilité de rétablir la paix sociale.

5 À partir des travaux des historiens de Coubertin, qui retracent son parcours intellectuel et politique et soulignent l'importance de son projet pédagogique (1), nous proposerons 
une généalogie de sa pensée. Cette démarche vise à souligner la permanence d'un questionnement éthique conservateur et libéral sur la dégradation des mœurs qui résulte de la destruction des anciennes institutions de l'Ancien Régime et du développement industriel sans nouvelles institutions démocratiques adaptées. Elle a pour but de mettre en lumière, à travers les écrits de Tocqueville et Le Play, la responsabilité de la classe industrielle, désormais dotée d'une liberté accrue, dans sa participation à l'intérêt général (2). Elle permet de retracer l'enchaînement qui, de l'analyse des institutions et des mœurs au lien social, conduit Coubertin à orchestrer une moralisation du capitalisme initiée par et pour les élites industrielles, guidée par des valeurs, anciennes dans leur forme, mais nouvelles dans leur contenu, les valeurs sportives (3). Il apparaîtra que la réforme pédagogique de Coubertin, destinée aux futures élites, est une solution pratique aux problèmes soulevés par ses prédécesseurs.

\section{Coubertin, sa pratique politique : influence et parcours}

6 L'histoire écrite par le Comité International Olympique et les différents ministères français des sports qui se sont succédés depuis la Seconde Guerre se souvient de Coubertin rénovateur des Jeux et père d'une doctrine, l'olympisme (Eyquem 1966, CNOSF 2006). Celle écrite par les historiens du sport et les tenants de la «théorie critique du sport $\|^{3}$, rappelle plus volontiers que Coubertin était un théoricien des élites, dont les écrits comme les actes témoignent de la nécessité de fabriquer, par la pédagogie sportive, une classe dirigeante à la fois virile et conquérante, morale et chevaleresque (Weber 1970-71, Boulongne 1975, Clastres 2005, Brohm 2008 pp. 27-29).

\subsection{Une figure du conservatisme libéral ralliée à la République}

7 Avant de devenir l'inventeur des Jeux Olympiques modernes (1894), l'action politique de Pierre de Coubertin était tournée vers la pédagogie sportive. Porté par l'esprit de la réforme sociale, forgé par une culture de l'Ancien Régime, mais rallié à la République, Coubertin se donne pour mission de transformer le système éducatif français en généralisant la pratique du sport dans les écoles. Son but est d'éduquer le jeune adolescent de la classe aisée, d'en faire un homme qui soit performant dans la société industrielle et surtout animé par des principes moraux (Brohm 2008 p. 45). Il accorde ce rôle social au sport : il l'estime apte à porter des bonnes valeurs chez les élites et in fine à construire une République libérale, protégée du risque révolutionnaire, des souvenirs de la défaite de 1870 et de la Commune (Clastres 2004). Ce projet social s'inscrit dans la continuité de l'œuvre de deux penseurs français qui ont contribué à sa formation intellectuelle et politique: Frédéric le Play et Alexis de Tocqueville (Boulongne 1975, Clastres 2005]. Quoique divergeant dans leur appartenance politique, Tocqueville, le républicain, et Le Play, le légitimiste, inscrivent leur réflexion dans une problématique commune, conservatrice et libérale - restaurer l'ordre social (Tréanton 1984, Castel 1999 p. 390, Jaume 2008 p. 86-91) - qui trouve ses fondements chez Burke et Montesquieu (Aron 1960, Dion 1967, Coronel de Boissezon 2007). Dans la lignée de ces historienssociologues, Coubertin entreprend de poursuivre l'analyse des mœurs et des institutions. Sur les traces de Tocqueville, Taine et Le Play, il effectue des voyages, étudie les mœurs, 
compare les sociétés et distingue les bonnes pratiques des mauvaises, en laissant toujours sur son passage un « arrière-goût de 'noblesse oblige'« (Weber 1970-71).

Coubertin fait partie de ceux qui ont décidé de participer à la construction de la République plutôt que de continuer à regretter l'Ancien Régime. Ce choix explique sa distance avec le milieu leplaysien et sa préférence pour « le vrai Le Play » qui diffère « de l'image posthume qu'en présentait ses disciples » (Coubertin 1931 cité par Clastres 2003). Son histoire avec le milieu leplaysien dure le temps d'une décennie ; elle commence avec la présentation de «L'éducation anglaise » en 1887 à la Société d'Économie Sociale fondée par Le Play et se termine avec la publication de son premier ouvrage, L'Évolution française sous la III République (1896). La critique qu'il y adresse aux catholiques français, qu'il juge responsables de l'anticléricalisme républicain, lui valut un compte-rendu anonyme et cinglant dans la revue leplaysienne qui motiva la rupture ; celle-ci faisait suite à plusieurs épisodes houleux avec la "clientèle cléricale» dérangée par sa posture œcuménique (Coubertin 1931 cité par Clastres 2003). Tocquevillien dans ses choix politiques, son alliance avec le républicain Jules Simon consacre la transition. En préservant le principe de continuité cher à Le Play, Coubertin choisit la République sans compromettre ses positions : il propose de faire du neuf - développer une «chevalerie sportive » - tout en retenant l'esprit de l'ancien - l'esprit chevaleresque - pour transformer les mœurs de la société et résoudre la question sociale.

\subsection{D'une pédagogie sportive au service des élites à l'olympisme}

9 En s'associant avec Jules Simon pour fonder le Comité pour la propagation des exercices physiques dans l'éducation en 1888, Coubertin est accusé d'avoir «commis un crime de lèse-majesté leplaysienne »: celui d'avoir recherché le soutien de l'État plutôt que de poursuivre dans la voie de l'initiative privée (Clastres \& Duval 2003 p. 23). Dix ans plus tard, alors que la bataille se livre entre l'Église et l'État sur l'espace laissé à l'initiative privée dans l'éducation, il se consacre à la réforme des lycées publics (Clastres \& Duval 2003). Il s'éloigne des monarchistes, nombreux dans la Société d'économie sociale, et cherche un compromis républicain qui se traduit par un rapprochement avec les organisations populaires. En fondant en 1906, «la société des sports populaires», mais sans attacher d'importance au fait que la jeunesse issue de milieux sociaux différents se rencontre dans la compétition sportive, il envisage d'étendre son action pédagogique audelà des futures élites.

Bien que ses choix apparaissent comme un infléchissement des "positions sportives élitaires ", Clastres estime que cette évolution relève davantage d'une stratégie politique que d'une mutation de sa pensée: si Coubertin «abaisse sa garde devant le flot démocratique, il n'abandonne pas pour autant tout idéal de patronage » (Clastres $2005 \mathrm{p}$. 291). Ainsi après avoir tenté, sans réel succès, de mettre en pratique ses idées pédagogiques par des actions de lobbying dans l'espace national, il emprunte la voie internationale pour poursuivre son projet de pacification en insistant davantage sur une participation ouverte à toutes les catégories sociales (Clastres 2005 p. 287). Bien que cette reconversion au «sport pour tous » soit entendue comme une "tension» de la pensée coubertinienne par Ferry (1997), comme une «imposture» par Brohm (2008), elle apparait surtout comme une autre manière de mettre en pratique l'idée leplaysienne de patronage (Clastres 2005). De la réforme sociale par la pédagogie à l'olympisme, Coubertin poursuit un projet pédagogique qui s'attache à répondre à un seul et même objectif, la 
paix, déclinée sous ses deux dimensions, la paix entre les classes, la paix entre les nations. En contribuant à la naissance du Comité International Olympique - institution internationale et privée - Coubertin assoit ses positions élitaires. Il participe à la construction des institutions qu'il estime bonnes, qu'elles soient nationales ou internationales. Il évolue d'une pédagogie sportive au service de la paix sociale, pensée par et pour les futures élites, à une pédagogie sportive, au service de la paix internationale, pensée par les élites pour la moitié de l'humanité, les hommes. «Révolté à l'intérieur de son milieu social », Coubertin fait ainsi le choix de moderniser « la tradition conservatrice "; il l'amende " de toute tendance cléricale et légitimiste, pour n'en retenir que les aspects pacifistes et internationalistes »(Weber 1970-71).

«L'olympisme est un renverseur de cloison. Il réclame l'air et la lumière pour tous. Il préconise une éducation sportive généralisée, accessible à tous, ourlée de vaillance virile et d'esprit chevaleresque, mêlée aux manifestations esthétiques et littéraires, servant de moteur à la vie nationale et de foyer à la vie civique. Voilà son programme idéal » (Coubertin 1919 cité par Attali \& Chapron 2004).

11 Cet extrait de la "Lettre olympique », publié dans La Gazette de Lausanne, souligne la continuité du projet social de Coubertin : transformer les mœurs en faisant renaître la "vaillance virile et l'esprit chevaleresque " qui manque à la société démocratique et industrielle, en péril par manque d'unité nationale. Ce "programme idéal » repose sur des fondations qui mobilisent l'histoire et la tradition; il hérite du problème éthique identifié par les conservateurs réformistes, la dégradation des mœurs, et d'une solution, l'édification de nouvelles institutions libres.

\section{La liberté privilège héritée de l'Ancien Régime comme levier de la responsabilité}

12 L'étude des mœurs fait écho chez Tocqueville et Le Play à deux auteurs de la fin du dixhuitième siècle, qui ont marqué la pensée européenne, Montesquieu et Burke. Au-delà des spécificités qui caractérisent leurs œuvres respectives, ces auteurs ont contribué à fonder en France le courant conservateur et libéral. Burke a rendu hommage à Montesquieu (Courtney 1963). Tocqueville, lecteur et admirateur de Burke, a su, comme le conseille Rémusat, l'analyser et le combattre (Lakoff 1998, Gannett 2003) en poursuivant l'œuvre de Montesquieu (Aron 1967 p. 226, Audier 2004 pp. 62-67). Enfin, Le Play a rappelé la contribution intellectuelle de chacun d'eux à sa pensée (Dion 1967, Coronel de Boissezon 2007).

\subsection{L'héritage de Burke et de Montesquieu : la disparition de l'esprit chevaleresque}

Les analyses tocquevilliennes et leplaysiennes sur l'évolution des mœurs font suite à la chute de l'Ancien Régime et au développement du commerce et de l'industrie. Elles résonnent particulièrement avec les deux thèses qui ont fait la postérité de L'Esprit des lois (1748) et des Réflexions sur la Révolution de France (1790): respectivement le «doux commerce » et la « disparition de l'esprit chevaleresque ».

La thèse de Montesquieu s'inscrit dans une analyse générale de l'influence des mœurs sur les lois et des lois sur les mœurs. Elle repose sur une observation: " partout où il $\mathrm{y}$ a des mœurs douces, il y a du commerce, et partout où il y a du commerce, il y a des mœurs 
douces » (Montesquieu 1748 II p. 9). Entendu comme un « rempart au despotisme », parce qu'il dispose d'une autonomie relative au regard du pouvoir du souverain, le commerce permet de «faire échouer la violence politique, l'arbitraire et l'abus de pouvoir » (Spector 2006 p. 175). Toutefois si Montesquieu valorise les lois du commerce parce qu'elles favorisent la diffusion des "mœurs douces", il rappelle également qu'elles corrompent «les mœurs pures ». Elles " perfectionnent les mœurs, par la même raison que ces mêmes lois perdent les mœurs » (Montesquieu 1748 II p. 9), si bien que l'esprit du commerce et l'amour du gain dominent désormais l'esprit chevaleresque et l'amour de la gloire (Hirschman 1977 pp. 14-15, Spector 2006 p. 177).

Dans les Réflexions sur la révolution de France, l'ouvrage fondateur du conservatisme politique européen, Edmond Burke poursuit l'analyse des mœurs initiée par Montesquieu et se focalise sur les institutions. Celles-ci servent la critique de l'universalisme de la Déclaration des Droits de l'homme et du citoyen de 1789. Définissant le droit comme le produit des anciennes institutions, comme le résultat d'une histoire singulière propre à chaque nation, Burke oppose les droits de l'homme de 1789, abstraits, aux «droits des Anglais ", hérités d'une histoire séculaire et de la tradition. À ce titre, il estime que le droit à la liberté se fonde sur la tradition. Il défend et regrette la liberté qui animait les anciennes institutions, parce qu'elles étaient les garantes de la permanence des mœurs chevaleresques. Cette chevalerie, "qui ennoblissait tout ce qu'elle touchait», qui «a donné son caractère à l'Europe moderne » et fait sa grandeur, a produit une «noble égalité » qui, transmise "à tous les niveaux de la vie sociale ", assurait «l'harmonie des différents aspects de la vie » (Burke 1790 p. 97). Il déplore l'avènement de la Révolution Française et le renversement des anciennes institutions qui ont contribué à la « ruine des mœurs et des opinions anciennes ", à l'extinction du « vieil esprit féodal et chevaleresque de loyauté » (Burke 1790 p. 99).

L'âge de la chevalerie est passé. Celui des économistes et des calculateurs lui a succédé; et la gloire de l'Europe est éteinte à jamais. Jamais, jamais plus nous ne verrons cette généreuse loyauté envers le rang et le sexe, cette soumission fière, cette digne obéissance, et cette subordination du cœur qui, jusque dans la servitude, conservait vivant l'esprit de liberté haute et grave. On ne connaîtra plus cette grâce spontanée de l'existence, cette générosité du cœur qui assurait librement la défense des peuples, tout ce qui nourrissait les sentiments virils et d'amour des entreprises héroïques. (Burke $1790 \mathrm{p} 96$ )

La dominance de l'intérêt et du calcul économique, la disparition de la noblesse du devoir et la perte des valeurs de l'Ancien Régime sont des craintes qui animent autant Tocqueville que Le Play. Ces derniers opposent toutefois à Burke une lecture moins critique du Nouveau Régime. D'un côté, Tocqueville apprécie dans les sociétés démocratiques la simplicité des rapports entre égaux et le processus d'adoucissement des mœurs qui développe «une compassion générale pour tous les membres de l'espèce humaine » (Tocqueville 1840 II pp. 208-209). De l'autre, Le Play apprécie « l'abrogation des régimes de contrainte [qui] a donné chez les modernes, une impulsion féconde aux vertus individuelles » (Le Play 1878 III p. 166) et reconnaît que « les sociétés prospères de notre temps [...] possèdent [...] en plus grand nombre que les sociétés anciennes, des individus habiles et prévoyants » (Le Play 1878 III p. 169). En mettant ainsi en valeur ces qualités, Tocqueville et Le Play élaborent les outils d'analyse nécessaires pour dépasser le regret de Burke. Ils fournissent à Coubertin les moyens d'envisager la possibilité d'ériger des nouvelles institutions adaptées aux démocraties marchandes et industrielles, qui puissent 
faire naître de nouvelles "entreprises héroïques » et nourrir les "sentiments virils » (Burke 1790).

\subsection{La « médiocrité » des mœurs de l'aristocratie industrielle}

Tocqueville analyse dans La Démocratie en Amérique les conséquences de l'égalisation croissante des conditions matérielles et étudie la transformation générale des sociétés qui résulte de la démocratisation. Il centre son analyse sur «l'égalité des conditions », le fait majeur de la démocratie américaine, en tant qu'il «crée des opinions, fait naitre des sentiments, suggère des usages et modifie tout ce qu'il ne produit pas " (Tocqueville 1835a I p. 57). Son travail s'appuie sur une hypothèse fondatrice: le fait d'avoir déclaré l'égalité comme un droit naturel lui a donné un caractère irréversible. Aussi le choix politique que Tocqueville présente à ses lecteurs ne se situe-t-il pas entre la démocratie et une nouvelle Restauration, mais plutôt entre une démocratie éclairée et une démocratie du désordre qui se confond avec la révolution et conduit à la tyrannie (Benoît 2000 p. 28). Il « déduit du fait démocratique la primauté du commerce et de l'industrie, l'élévation du niveau de vie » (Aron 1960) et établit un «lien étroit », un « rapport nécessaire » entre « liberté et industrie » (Tocqueville 1840 II p. 175).

L'analyse comparée des mœurs amène Tocqueville à montrer que le modèle d'organisation démocratique fabrique un individu replié sur ses intérêts privés, peu soucieux du bien général. Ce modèle laisse la place à un nouveau despotisme, celui de l'intérêt privé, avec pour seul but d'accroître le bien-être matériel des individus. Les hommes « contractent plus ou moins les habitudes intellectuelles des classes industrielles et commerçantes; leur esprit prend un tour sérieux, calculateur et positif»; ils se détournent « de l'idéal pour se diriger vers quelque but visible et prochain qui se présente comme le naturel et nécessaire objet des désirs » (Tocqueville 1840 II p. 259). «L'esprit d'entreprise l'emporte [...] Le désir de richesse prend la place du désir de gloire et de l'ambition" (Aron 1962 p. 39). L'expérience de l'activité industrielle et commerciale transforme alors les habitudes et les opinions. Celles-ci influencent les institutions, lesquelles conduisent à l'effondrement des valeurs et à la disparition du lien social qui prévalait dans l'Ancien Régime (Manent 2001 p. 184-187). Tocqueville ne masque pas son intérêt pour ce qu'il observe en Amérique. S'il adopte le principe de liberté-égalité et se positionne en faveur de l'instauration d'une démocratie marchande, il en dénonce les effets pernicieux : la paupérisation de la classe laborieuse et la déresponsabilisation de la classe industrielle.

Dans le Mémoire sur le paupérisme (1835b), Tocqueville étudie les causes de ce qu'il nomme «fléau social » et conclut que ni les industriels ni les travailleurs ne doivent compter sur la charité publique, parce qu'elle déresponsabilise les premiers qui manquent à leur devoir de protection et les seconds qui manquent à leur devoir de prévoyance. Cette critique fait écho à la seconde Démocratie, dans laquelle il porte son analyse sur « l'aristocratie industrielle ». Cette classe, qui émerge du fait même de la démocratie, n'a «pas d'esprit ni d'objets communs »; elle ne constitue pas un « corps » (Tocqueville 1840 II p. 200). Contrairement à l'aristocratie de l'Ancien Régime, elle n'unit pas ses membres, elle n'établit pas non plus de "lien véritable entre le pauvre et le riche" (Tocqueville 1840 II p. 201), « ni par l'habitude, ni par le devoir.» (Tocqueville 1840 II p. 202). "Médiocre", cette aristocratie n'a pas d'histoire (Aron 1962 p. 42). Alors que «l'aristocratie territoriale des siècles passés » était obligée "par la loi » ou "par les 
mœurs ", de secourir « ses serviteurs ", « l'aristocratie manufacturière [...] appauvri[t] et abruti[t] les hommes dont elle se sert [et] les livre en temps de crise à la charité publique pour les nourrir» (Tocqueville 1840 II p. 202). Observant que cette aristocratie est «la plus dure qui ait paru sur terre », quoiqu'elle reste pour l'heure " la plus restreinte et la moins dangereuse» (Tocqueville 1840 II p. 202), Tocqueville considère «la classe industrielle comme ayant reçu de Dieu la mission spéciale et dangereuse de pourvoir à ses risques et périls au bonheur matériel de toutes les autres. » (Tocqueville 1835b p. 12)

Méfiant comme Tocqueville à l'égard des aristocraties de l'Ancien Régime, qui ont contribué à la "décadence graduelle des institutions propres du Moyen Age » (Tocqueville 1858 p. 11), Le Play estime qu'elles ont manqué à leur devoir de protection. Il les juge responsables de la destruction des anciennes institutions et, avec elle, de la dégradation des mœurs et du paupérisme (Le Play 1878 III pp. 154-157). Pour asseoir sa thèse, il adopte une démarche empirique et analyse la formation des mœurs. Il considère d'abord la famille comme «l'unité sociale » par excellence et l'érige, avec la religion et la propriété, en « institution immuable » qui prolonge et perpétue l'individu père de famille (Le Play 1878 II p.6). Puis il observe et compare: comme Tocqueville, Le Play craint l'instabilité française et admire la fermeté et la stabilité des "situations privées et des pouvoirs publics » en Angleterre (Le Play 1878 I p. 13). Afin de résoudre la question du paupérisme source d'antagonisme de classes, Le Play distingue «les œuvres tendant à pallier les souffrances journalières, des institutions ayant pour effet de guérir radicalement le mal et d'en prévenir l'explosion » (Le Play 1878 III p. 167). Il se centre sur les secondes, considérant que «la pauvreté n'a pas cessé de se propager [...] à mesure que les institutions féodales étaient abrogées dans l'Occident» (Le Play 1878 III p. 156). Comme Tocqueville, il dénonce le caractère momentané des contrats; il regrette la permanence des rapports que l'Ancien Régime entretenait entre les classes, et centre son analyse sur la destruction du lien social engendrée par l'échange contractuel, l'industrialisation et l'absence d'institution. Dénonçant ce régime qui pèse «sur des individus brusquement arrachés à un antique patronage » (Le Play 1878 III p. 159) et qui engendre le paupérisme, Le Play renvoie la classe laborieuse et la classe possédante à leur responsabilité respective. Comme Tocqueville, il fait peser sur les industriels une responsabilité plus lourde, parce qu'ils profitent d'une nouvelle liberté sans en assumer les devoirs. Comme Tocqueville, il estime que la réforme doit résulter d'abord de leur initiative, par l'instauration du patronage, l'institution par excellence capable d'éradiquer le paupérisme.

\subsection{De la liberté, héritée de l'Ancien Régime, au devoir de patronage}

21 Au cœur du modèle d'organisation qui supprime les classes sociales de l'Ancien Régime, se pose la question de la noblesse qui incombe aux statuts économiques du maître face à l'ouvrier. Cette question est, selon d'Iribarne, indissociable de la conception de la liberté qui prévaut chez Tocqueville. Elle est par-là même pour l'ethno-sociologue de l'entreprise indissociable de l'analyse des formes culturelles de management ${ }^{4}$. L'intérêt affiché de Tocqueville pour la liberté venue d'Amérique pourrait laisser croire qu'il s'est converti à la vision anglo-saxonne de la liberté (d'Iribarne 2003 p. 971). Cependant sa volonté d'opposer une conception démocratique à une conception aristocratique de la liberté n'a pas conduit l'auteur de la Démocratie à s'y convertir entièrement. Si parfois il rejoint la vision lockéenne d'une liberté qui relève d'un droit du propriétaire, Tocqueville reste fidèle à la conception de la liberté qui prévalait dans l'ordre aristocratique : celle qui, 
fondée sur le privilège, implique devoir et responsabilité (d'Iribarne 2003 p. 972). Dans la continuité de son travail de terrain analysé dans La Logique de l'honneur (1989), d'Iribarne rappelle que «les exigences de l'honneur, telles qu'elles sont décrites par Montesquieu, n'ont pas cessé d'être de mise " (d'Iribarne 2003 p. 975). Ainsi, le regard porté sur la démocratie en Amérique permet à Tocqueville d'identifier les limites d'un modèle contractuel où l'intérêt bien compris et le calcul coût/avantage conduisent à l'effacement « de la beauté du geste » (Guellec 1996 p. 32). Il laisse à ses héritiers le devoir de doter la France "d'institutions adaptées" pour se mettre à l'abri du vent révolutionnaire qui entretient les braises de la division (Guellec 1996 p. 36).

La réflexion de Le Play répond également au souci de dépasser la simple référence à la liberté contractuelle. Considérant que l'accroissement de la liberté individuelle est la raison pour laquelle "les sociétés modernes l'emportent sur les sociétés anciennes", parce que « les peuples en devenant plus libres et plus prospères, substituent l'initiative des citoyens à l'intervention des fonctionnaires publics » (Le Play 1878 II p. 78), Le Play reconnaît la supériorité du nouveau régime qui libère des individus et offre des opportunités de développement des talents. Il reproche toutefois « aux anglais » d'avoir poussé « la liberté individuelle jusqu'à ses extrêmes limites », et d'avoir " cru améliorer le travail manufacturier en restreignant le cercle des devoirs sociaux» (Le Play 1878 III p. 164). Mal utilisée quand elle est fondée sur des "engagements momentanés », cette liberté dont profite la classe «supérieure » engendre le « mal social»- le paupérisme et l'antagonisme de classes (Le Play 1878 III p. 175). Il met en garde contre la « corruption du corps social » que le progrès de la richesse pourrait engendrer sans « une pratique plus assidue des lois morales» (Le Play 1878 I p. 11). Soucieux d'unir les "patrons et les ouvriers " qui «croient avoir des intérêts opposés ", il cherche à instituer un nouvel ordre des récompenses, (Le Play 1878 I p. 6). Ce faisant, il accorde un rôle central à l'initiative privée, et l'assoit sur une conception de la liberté qui s'inscrit dans la continuité de l'Ancien Régime. Sa réforme s'inspire des rapports de protection qui assuraient «la continuité » entre le seigneur et ses vassaux (Le Play 1878 III p. 176). Nouvelle, imprégnée d'Ancien Régime et de catholicisme, cette liberté accorde à la classe dirigeante la mission - «de droit divin» - de réaliser la réforme sociale (Dion 1967). Entendue comme un privilège, elle implique d'instaurer un nouvel ordre de récompense fondé sur le "régime des engagements volontaires permanents». Ce nouvel ordre, nommé patronage, est la «nouvelle organisation, dans laquelle les maîtres et les seigneurs de l'ancien régime sont remplacés par des patrons. » (Le Play 1878 III p. 174). Il se reconnaît à la " permanence de rapports maintenue par un ferme sentiment d'intérêts et de devoirs réciproques » (Le Play 1878 III p. 175).

Tocqueville, Le Play, et Coubertin avec eux, appartiennent ainsi à ce courant libéral critique vis-à-vis de l'économie politique anglaise qui marque la fin du «libéralisme utopique » (Castel 1999 pp. 388-390). Anti-malthusien, ce courant ne pense pas éradiquer le paupérisme par un laisser-faire qui proscrirait tout type de charité, à l'image de celui proposé par Bastiat (Castel 1999 p. 391). En affichant la primauté de la liberté de ceux qui ont le privilège de commander le travail, au détriment d'une intervention de l'État, ce courant adopte une "stratégie de moralisation" de la société industrielle destinée à contrer les socialistes français et à contrôler les «classes laborieuses, classes dangereuses » (Castel 1999 p. 396). Aussi, plutôt que de laisser-faire le marché, ce courant préconise-t-il de laisser-faire «les citoyens éclairés » guidés par le devoir plutôt que le calcul et l'intérêt bien compris (Castel 1999 p. 390). Cette démarche résonne avec le 
renouveau libéral qui se dessine depuis la fin du XXe siècle comme une alternative au compromis keyneso-fordien des Trente Glorieuses : il s'agit de "théoriser l'intégration des processus éthiques dans la science économique et [de] poser les fondements d'une éthique compatible avec l'économie de marché et un libéralisme qui ne serait plus tout à fait guidé par le 'main invisible' tout en évitant de recourir aux régulations étatiques » (Salmon 2002 p. 87). L'intégration des processus éthiques se traduit chez Tocqueville et Le Play par une transformation des pratiques de la classe industrielle; elle se théorise à travers une analyse des institutions, les bonnes - les institutions libres (Tocqueville 1840 II p. 131).

\subsection{L'association, l'institution libre des démocraties marchandes}

24 L'édification des bonnes institutions qui accompagnent la démocratie marchande est motivée chez Tocqueville par la nécessité de se mettre à l'abri du «despotisme de la majorité » qui trouve son expression dans l'intervention de l'État, et de veiller à sauvegarder la liberté en érigeant des institutions adaptées (Aron 1967 p. 227- 230, Audier 2004 pp. 65-66). Contre Rousseau qui affiche la nécessité de protéger l'intérêt général des intérêts privés exprimés par de multiples groupes de pression (Benoît 2004 pp. 85-89), Tocqueville poursuit la réflexion de Montesquieu sur le despotisme des monarchies et l'applique à la démocratie. Il hérite du choix de l'auteur de L'Esprit des lois entre la liberté des monarchies modernes fondée sur l'honneur et l'inégalité des conditions, la liberté des petites républiques fondée sur la vertu et l'égalité (Aron 1960, 1967). La voie qu'emprunte Tocqueville, destinée à dépasser ce choix, fonde la liberté sur l'égalité des conditions ; mais l'expérience américaine qui esquisse la possibilité d'une république fondée sur l'intérêt, ne satisfait pas entièrement l'observateur français. Car «Tocqueville n'est nullement un admirateur béat de la société américaine » qui favorise un «bien-être sans éclat ni grandeur » (Aron 1967 p. 238). De la première Démocratie à la seconde, puis de la seconde à L'Ancien Régime et la révolution (1858), nombreux sont les lecteurs de Tocqueville qui soulignent la distance graduelle que l'auteur prend vis-à-vis de l'Amérique utilitaire, pour revenir à une conception aristocratique des institutions démocratiques (Aron 1960, 1967, Guellec 1996, Benoît 2004). Ces institutions sont destinées à « préparer les hommes à être libres» (Tocqueville 1835a I p.400), à pallier «le manque d'accoutumance à la pratique de la liberté » et pour in fine sauvegarder la liberté de la tyrannie de la majorité (Aron 1960). L'accent alors est mis sur les associations civiles (Tocqueville 1840 II p. 137).

Tocqueville voit dans l'association l'institution démocratique capable de jouer le rôle que tenait « le grand seigneur » dans les sociétés aristocratiques. Il l'estime apte à conduire des "grandes entreprises", "faire isolément des grandes choses » (Tocqueville 1840 II pp.137-138). Le regard qu'il porte sur le monde associatif américain le conduit à reconnaître l'efficacité et le pouvoir du calcul raisonnable et éclairé, notamment dans sa capacité à réduire le paupérisme (Guellec 1996 p. 32, Ferraton 2004) ; mais sa prescription ne prend pas le chemin du modèle américain (Tocqueville 1840 II, p. 324, Aron 1967 p. 239). En accordant sa préférence à la grandeur et la beauté du geste, Tocqueville reste attaché aux regrettées «entreprises héroïques » de Burke, qu'inspiraient les anciennes institutions. Aussi l'association tocquevillienne prend-elle ses distances avec le modèle utilitaire et s'oriente alors vers un renouveau "démocratico-aristocratique " (Chanial 2004) : elle devient le moyen pour la démocratie marchande d'assurer la survivance de la 
liberté aristocratique et des valeurs qui l'accompagnent, et par-là même, de résoudre la question sociale.

Le souci de mettre en place des processus d'apprentissage de la liberté - comprendre que cette liberté privilège implique des devoirs - conduit Tocqueville et Le Play à poursuivre leur réflexion vers l'éducation et les institutions qui s'y rattachent, la famille, la religion et l'État. Tocqueville n'a pas consacré de chapitre exclusif à cette thématique dans la Démocratie, mis à part celui intitulé « De l'éducation des jeunes filles aux États-Unis » dans la seconde (Tocqueville 1840 II pp.147-149). Néanmoins son ouvrage regorge de métaphores scolaires (Guellec 1996, Chabot 1996). L'évolution des mœurs étant inséparable de la manière de transmettre les pratiques, l'éducation devient une notion centrale, évidente, permanente de sa pensée. Prenant exemple sur les États-Unis où l'enseignement est pris en charge par des multiples clergés, et non par un clergé apparenté à une religion d'État, il se positionne en faveur de la liberté de choix des parents, de la diversité des méthodes pédagogiques et de la mise en concurrence des établissements scolaires (Chabot 1996).

Le Play s'est plus explicitement consacré à cette question. Dans le même esprit que $L a$ Démocratie, les parties de La Réforme sociale consacrées à la religion (livre I) et à la famille (livre II) traitent pour l'essentiel d'éducation. À cela s'ajoute le chapitre spécifiquement dédié à cette thématique dans le Livre $\mathrm{V}$ consacré aux associations, dont le titre illustre bien sa priorité : "La grande fonction des corporations libres est l'enseignement supérieur des sciences des lettres et des arts » (Le Play 1878 III pp. 54-125). Il se positionne en faveur du modèle anglais où la gestion des écoles, payantes, est assurée par les familles et les anciens élèves, et financée par des legs et des dons. Enfin, concernant la promotion et la diffusion du patronage volontaire, Le Play estimait que l'éducation des futures " classes dirigeantes » dans cette direction, réalisée au sein des « corporations libres", suffirait à « guider » et à transformer les classes laborieuses et les peuples colonisés, par la diffusion des bonnes pratiques (Le Play 1878 III pp. 167-200, Dion 1967).

\section{La Réforme Sociale par une pédagogie sportive}

Coubertin a écrit de nombreux ouvrages qui vont des travaux historiques aux monographies, des articles de pédagogie sociale aux analyses des gestes sportifs (Boulongne 1975 pp. 395-430). Sans frontière disciplinaire claire, la diversité de ses travaux en fait un héritier de Le Play et Tocqueville à plusieurs titres. D'abord, Coubertin poursuit les études comparées des sociétés occidentales. Ensuite, soucieux de contribuer à la résolution de la question sociale, il met en application leurs idées: il s'attache à développer les mécanismes d'apprentissage de la liberté - une liberté responsable, entendue comme un privilège - et s'intéresse au rôle des institutions éducatives. Enfin, dans sa pratique politique, il participe à l'édification des nouvelles institutions : elle débute par la fondation du Comité pour la propagation des exercices physiques dans l'éducation (1888), se poursuit par sa tentative de réforme des lycées français (1890) et la formation du Comité International Olympique (1894); elle s'achève, avec Laville, historien et membre du Parti Colonial, par la naissance d'un établissement scolaire, le Collège de Normandie (1901), concurrent de l'École des Roches (1898) fondée par deux leplaysiens, Demolins et Tourville, à Verneuil-sur-Avre (Clastres \& Duval 2003). Disciple à l'écoute des prescriptions de ses prédécesseurs, Coubertin, historien-sociologue, se fait théoricien de la pédagogie et praticien. 


\subsection{L'exemple de l'Angleterre, Arnold et la muscular christianity} anglais. Lecteur des Notes sur l'Angleterre (1872) de Hyppolite Taine, il poursuit le travail entrepris sur l'éducation en se centrant sur l'apprentissage de la liberté et du selfgovernment (Boulongne 1975 p. 114). En novembre 1886, âgé de 23 ans, il publie son premier article, dans la revue leplaysienne, La Réforme Sociale: "Les collèges anglais, Harrow School ». En avril de l'année suivante, une fois son étude nourrie de trois autres collèges, il présente ses premières conclusions devant la Société d'économie sociale dans «L'éducation anglaise » (Coubertin 1887). Son travail de terrain est appuyé par les écrits pédagogiques de Thomas Arnold, Headmaster de l'École de Rugby qui fut une figure importante du mouvement initié par Charles Kingsley et Thomas Hughes: la muscular christianity (Boulongne 1975 p 120, Putney 2003 pp. 12-14). Arnold, que Coubertin considère comme « le père de l'éducation anglaise actuelle » (Coubertin 1887), se propose de former des Christian Gentlemen : «mon but est d'apprendre aux enfants à se gouverner eux-mêmes [...] [plutôt] que de les gouverner bien moi-même» (Arnold, cité par Coubertin 1887 p. 127). Il importe à ses yeux que la jeunesse qui compose «les futurs maîtres » apprenne «à connaître sa puissance » pour la mettre au service «le plus tôt possible » avec « la responsabilité lourde qui est le contrepoids de tout pouvoir » (Arnold, cité par Coubertin 1887 p. 128).

[Les muscular christians] ont hérité de la vieille maxime chevaleresque que le corps de l'homme doit être bien exercé et développé par son maître pour ensuite servir à la protection des plus faibles, à l'avancement de toutes les causes justes et à la conquête du monde (Arnold, cité par Coubertin 1887 pp. 136-137)

"L'apprentissage de la liberté ", idée chère à Tocqueville comme à Le Play, est l'élément central de la pédagogie d'Arnold. «La liberté et le sport [...], ces deux mots renferment à eux seuls tout le système" (Coubertin 1887 p. 139) : le jeune Coubertin salue par ces termes la muscular christianity dont le mérite est d'avoir trouvé le moyen de déployer les qualités "physiques, morales et sociales» nécessaires aux futures élites, libres et responsables (Coubertin 1887 p. 135). Il retient également les vertus de l'association et de la pratique de l'organisation sportive: «Les jeux sont un terrain parfait d'éducation sociale [...] [les élèves] cotisent, ils élisent leur chef, et leur obéissent ensuite avec un remarquable esprit de discipline » : ils gèrent et se coordonnent «dans un embryon de société » (Coubertin 1887 p. 139). «Les comparaisons que suggérait le voyage » font «pense[r] presque involontairement à la France» (Coubertin 1888 p. 289); elles permettent à Coubertin d'identifier les carences du système éducatif français « où les bonnes volontés s'arrêtent en chemin, faute de ressort" (Coubertin 1887 p. 139). «L'amollissement des mœurs, le souvenir de la défaite de 1870, et la décadence des élites ", à l'origine du retard français, tant du point de vue du développement industriel que de la conquête coloniale, assoient sa critique (Boulongne 1975 p. 245). Il dénote l'impuissance de sa classe qui ne sait plus diriger et de sa nation qui conduit à la « médiocrité générale » (Coubertin 1888 p. 306, Boulongne 1975 p. 84).

\subsection{Préparer les élites à diriger la démocratie}

Les travaux ultérieurs de Coubertin, qui portent sur pédagogie sportive, sont rassemblés dans Essais de psychologie sportive (1913) et Pédagogie sportive (1920). Ils témoignent de la

Économie et institutions, 20-21 | 2014 
continuité de son projet éducatif et confirment que sa pensée s'inscrit dans une réflexion globale sur la société industrielle et le nouveau régime. Mener la Réforme sociale consiste à ses yeux à "opérer des changements en répondant aux besoins nouveaux » qui s'imposent pour s'accorder avec le «règne des principes démocratiques » et "l'égalitarisme relatif» (Coubertin 1913 p. 27). Il s'agit de "préparer " la démocratie libérale, en trouvant l'équilibre qu'il juge nécessaire entre concurrence et coopération.

L'État démocratique ne peut vivre et prospérer sans [un] mélange d'entr'aide et de concurrence [..]. Point d'entr'aide et l'on verse dans un individualisme brutal qui mène à l'anarchie; point de concurrence et c'est l'affaiblissement des énergies conduisant à la somnolence et à l'abdication (Coubertin 1920 p. 140)

La " coopération sportive » développée dans les sports d'équipe est ainsi vue comme une «école préparatoire à la démocratie " (Coubertin 1920 p. 140, Boulongne 1975 p. 140). Cette pédagogie est portée par la réalisation d'un idéal, «le retour à la vie grecque » (Coubertin fév. 1907 in 1913 pp. 27-33). Entendu comme un renouveau pensé dans le cadre des sociétés modernes, ce retour se présente comme une réponse à l'homme " tenté de se rebeller " contre l'existence "anti-humaine " provoquée par les mutations du travail conséquences de l'industrialisation. S'il décrit dans l'article consacré aux "sanatoriums pour bien-portants" (Coubertin avr. 1907 in 1913 pp. 41-50), la forme « saine » de travail, relativement nouvelle, qui récolte les fruits du progrès technique et bénéficie de la "réduction de la tâche quotidienne en durée et en intensité d'effort ", cette forme de travail qui « s'exer[ce] en plein air » reste «très rare » (Coubertin $1913 \mathrm{p}$. 42). Sans insister sur les effets néfastes de l'industrialisation sur le travailleur manuel, il centre son attention sur les méfaits du travail intellectuel. Les " métiers cérébraux » font subir aux travailleurs "une surchauffe incessante»; cette "pression mentale, assez exagérée pour devenir dangereuse " met l'homme en "situation de déséquilibre " (Coubertin 1913 p. 48). Dans «Le sport peut-il lutter contre la névrose universelle?» (Coubertin 1910 in 1913 pp. 119-131), il oppose « l'activité moderne avec ses imprévus, ses audaces, ses sursauts » (p. 121) à la « stabilité sociale » d'autrefois. Il y associe un «mal moderne ", qui ne frappe plus seulement les «faibles» de la société mais également les «forts » : la " névrose universelle ", phénomène moderne qui conduit à la "disparition du sentiment viril ». Il voit le sport comme le remède capable de «le faire renaître et l'entretenir » (Coubertin 1913 p. 127), de faire revivre «le goût de la force", une force " cultivée, travaillée, contrôlée et honnêtement utilisée » (Coubertin 1920 p. 137). Dans ces conditions, il «va de soi » pour Coubertin que cette éducation ne convient pas aux jeunes filles (Coubertin 1901 cité par Boulongne 1975 p. 222).

\subsection{De la gymnastique utilitaire au sport chevaleresque}

Dans «Le sport et la morale» (fév. 1910 in 1913 pp. 100-108), Coubertin construit son argumentation en discutant les propos d'une brochure sur "l'éducation virile et les devoirs physiques » qui se propose «d'employer tous les moyens à développer les qualités physiques » (Coubertin 1913 p. 100). Cette maxime revêt une forte résonance «utilitaire " qu'il conteste (Coubertin 1913 p. 101). Car, s'il estime que les exercices physiques peuvent contribuer à l'intérêt général, ils peuvent également servir toutes sortes de causes : «l'utilitarisme, l'altruisme, [et] l'égotisme ».

L'incertitude et l'effacement relatif des idées religieuses laissent la place libre à des cultes nouveaux ou renouvelés. Le plus naturel à l'homme quand il se détourne de Dieu, n'est-il pas le culte de soi-même? (Coubertin 1913 p. 103-104) 
Coubertin met ainsi en garde contre «le culte du corps » qui risquerait d'engendrer un «monstre sportif» (Coubertin 1913 p. 103-104), et contre « une initiation fallacieuse à la seule efficacité technique » (Boulongne 1975 p. 23). Pour s'en protéger, il juge nécessaire de reformuler la maxime proposée, en distinguant le caractère de la morale. Car si le sport développe «le courage, l'énergie, la volonté, la persévérance, l'endurance », ces qualités qui relèvent du caractère ne renseignent pas sur leur contenu moral. Conscient qu'il n'y a pas de «moralisation directe par le développement physique » (Coubertin 1913 p. 104-105), Coubertin amende la formule et propose « d'employer tous les moyens propres à développer nos qualités physiques pour les faire servir au bien collectif ». En ce sens, le sport « adjuvant de la morale », est mis au service « d'un but réfléchi de solidarité qui élève [l'homme] au-dessus de lui-même » (Coubertin 1913 pp. 105-106). C'est à cette condition, ajoute-t-il, que la Réforme Sociale sera possible, que la transformation des mœurs prendra la bonne direction. Le sport, ainsi entendu par Coubertin, ne se limite pas à une technique au service d'un corps destiné à augmenter le coefficient de capacité musculaire (Boulongne 1975 p. 218-220). Elevé au rang de « religion avec église, dogmes, culte ", il est destiné à devenir une institution qui favorise le maintien du "sentiment religieux » (Coubertin 1931 p. 103, Brohm 2008 pp. 30-32). Sa critique de la gymnastique utilitaire imprégnée des thèses hygiénistes, et sa préférence pour la chrétienté musculaire venue d'Angleterre à celle, venue des Etats Unis fondée sur l'intérêt, témoignent ainsi de sa volonté de construire une pédagogie mise au service de l'intérêt général et non seulement du bien-être de l'individu, de la productivité ou du profit (Boulongne 1975 pp. 207-217). Car, il importe autant pour Coubertin d'accompagner le développement de la société industrielle, afin de la rendre plus performante, que de trouver une " parade »à cette organisation sociale qui conduit à l'égoïsme, au repli sur soi et à la division sociale (Weber 1970).

"La saine sportivité d'une jeune génération prépare les succès nationaux » (Coubertin 1920, p. 139). Cette préparation à l'avenir s'élabore autour d'une réflexion sur la guerre et la paix, car aux yeux du pédagogue, l'esprit chevaleresque trouve sa source dans l'esprit militaire. Dans «Le sport et la guerre » (Coubertin avril 1912 in 1913 pp. 194-198), il souligne que la pratique du sport et de la guerre satisfont un même « instinct masculin » qu'il convient d'entretenir et surtout de guider vers le bien commun. D'un côté, il estime que la pratique du sport a une bonne influence sur l'esprit militaire parce qu'elle réduit l'esprit belliqueux: «La préparation indirecte à la guerre que comporte le sport " (Coubertin 1913 p. 194) fait qu'une " armée de sportsmen sera plus humaine, plus pitoyable dans la lutte, plus calme et plus douce après» (Coubertin 1913 p. 196). De l'autre, il montre que cette pratique renforce l'unité nationale, participe à la construction d'une démocratie libérale pacifiée par le commerce en contribuant à la renaissance des « mœurs pures » chères à Montesquieu. Ainsi le sport érigé en institution participe à la formation des nouvelles générations de possédants à l'esprit de conquête ; la puissance «des forts» est mise au service de la protection des «faibles» et de la grandeur de la nation.

\subsection{Discipliner les classes laborieuses et les peuples indigènes}

Les quelques écrits tardifs de pédagogie sportive à l'usage des classes laborieuses fournissent des éléments permettant de compléter le tableau de l'idéal moral de Coubertin. "La colère " analysée à de nombreuses reprises se retrouve au cœur de la 
définition - et de la résolution - de la question sociale : « qu'est-ce que la question sociale [...] sinon le produit d'une agglomération des 'ressorts tendus par la colère'?» (Coubertin 1920 p. 145). Source de tous les dangers, « la colère est partout dans le monde : elle trouble à la fois le foyer familial et les institutions sociales; elle compromet à la fois le repos de l'individu et la paix publique " (Coubertin 1920 p. 134). La colère - des hommes des classes laborieuses - résonne ici avec l'antagonisme social, déploré par Le Play, et le spectre de la Commune. "Le sport est le plus grand 'apaiseur' qui soit» (Coubertin 1920 p. 134). Coubertin rappelle à ce titre que l'ouverture de «salles gratuites de boxes dans les quartiers mal famés» par Théodore Roosevelt alors chargé de la juridiction de la police de New York, a contribué à une réduction considérable « des rixes sanglantes » (Coubertin 1920 p. 134). Toutefois si le sport apparaît comme un instrument de pacification sociale destinée à calmer la "colère diffuse contre les injustices " (Coubertin 1920 p. 133), sa pratique n'a pas pour but de favoriser la rencontre des différents milieux sociaux :

Que la jeunesse bourgeoise et la jeunesse prolétarienne s'abreuvent à la même source de joie musculaire, voilà l'essentiel; qu'elle se rencontre, ce n'est, présentement qu'accessoire. De cette source découlera, pour l'une comme pour l'autre, la bonne humeur sociale : seul état qui puisse autoriser pour l'avenir l'espoir de collaboration efficace (Coubertin 1920, p. 145)

Dans « Le sport et la colonisation » (Coubertin jan. 1912 in 1913 pp. 175-181), Coubertin soulève la question de la participation des peuples «indigènes » aux compétitions organisées par les colons. L'argument avancé est analogue à celui proposé en faveur des classes populaires. "Les sports sont un instrument vigoureux de disciplinarisation " (Coubertin 1913 p. 178). Ils permettent le développement de "bonnes qualités sociales » "de l'hygiène et de la propreté, de l'ordre et du self contrôle ». Tant que ce sont des «jeux populaires", que les "indigènes" «s'amusent», ils peuvent devenir "plus maniables qu'autrement ». Toutefois, il préconise de veiller à « ne pas leur laisser prendre des apparences trop militaires [...] qui pourrai[ent] les aider à préparer quelque rébellion future » (Coubertin 1913 p. 178). À ce titre, il rappelle qu'« il est extrêmement évident que la propagation du jiu-jitsu n'est pas désirable du point de vue de la domination européenne » (Coubertin 1913 p. 179). Ainsi le regard que porte Coubertin sur les classes populaires et "les indigènes" confirme ses positions élitaires et met en lumière une pédagogie différenciée à l'usage des différentes classes de la société :

- La première, mise au service des élites, est destinée à l'apprentissage du self-government et de la liberté.

- La seconde, mise au service des classes populaires et des peuples colonisés, est destinée à l'apprentissage du self-control, de l'obéissance et de la discipline.

Cette distinction induit deux catégories de bon comportement, et donc deux catégories d'institutions éducatives permettant de contribuer à la réalisation de la paix sociale et de l'unité nationale. La pédagogie différenciée et la pratique séparée sont un moyen pour Coubertin d'assurer la coexistence d'une bonne concurrence - qui entretient l'esprit de conquête et de liberté des élites - et d'une bonne collaboration - qui favorise l'adhésion et le contrôle des travailleurs nationaux et indigènes - indispensable à la stabilité des démocraties conquérantes et marchandes. 


\section{Conclusion}

39 Au regard des écrits et des actions politiques de Coubertin, il apparait que la thèse de Barbusse selon laquelle "à l'origine » sport et entreprise relèvent de mondes séparés perd de son sens. Cette thèse présume que le sport en tant qu'institution pédagogique a été pensé et développé, indépendamment d'une réflexion sur l'activité industrielle de production, pour se rapprocher à la fin du vingtième siècle de la culture d'entreprise. Elle suppose que ces deux mondes se sont retrouvés sur des principes et des pratiques qu'ils ont en commun dans le post-fordisme. Le retour aux sources a justement mis en lumière un processus bien différent : dès l'origine, le sport en tant que pédagogie a été pensé par Coubertin pour servir la démocratie libérale, pour donner à l'activité industrielle et commerciale, la noblesse qui lui manque, et pour transmettre la grandeur désintéressée, le sens de l'honneur, qui fait défaut à la nouvelle aristocratie. Il n'est donc pas contradictoire qu'au tournant du XXI ${ }^{\mathrm{e}}$ siècle les entreprises aient mobilisé les valeurs sportives pour redorer leur blason.

Afin d'opposer une thèse alternative à celle des "logiques convergentes ", nous avons ainsi esquissé une généalogie de la pensée de Coubertin. Cette pensée se forge sur le rejet de la logique de l'intérêt bien compris et puise sa conception de la liberté et de la responsabilité dans les valeurs de l'Ancien Régime. Nous avons montré que cette liberté responsable trouve ses racines chez Montesquieu et Burke, et se construit avec Tocqueville et Le Play. Ni vertueuse, ni utilitaire, elle est portée par la «logique de l'honneur » qui, de la théorie à la pratique, lègue aux successeurs de Tocqueville et Le Play le soin d'ériger les institutions adaptées aux démocraties marchandes. Ces institutions - libres - ont pour vocation de transformer les mœurs des classes dirigeantes, et par diffusion graduelle, de transformer celles de l'ensemble de la société. Coubertin, en lecteur attentif des thèses tocquevillienne et leplaysienne, prend cette mission à la lettre ; il propose une solution conservatrice et innovante, une réforme sociale par la pédagogie sportive.

41 De ce point de vue, cette pédagogie au service des élites va au-delà de l'intention de former des futurs dirigeants valeureux et efficaces ; elle s'inscrit dans un projet de société qui vise à la transformation des mœurs, à faire renaître l'esprit public qui caractérisait l'aristocratie de l'Ancien Régime, en s'appuyant sur la logique de l'honneur. Cette éducation différenciée, conçue comme une méthode d'apprentissage à la liberté et au selfgovernment, vise à combler le vide moral laissé par la démocratie marchande et industrielle et la destruction des anciennes institutions. Elle vise à empêcher la démocratie marchande de réduire ses pratiques sociales à la seule satisfaction de l'intérêt privé. Elle se propose d'ériger le sport en institution, tissée par un réseau associatif et un système éducatif où les fils de la classe dirigeante pourraient dans le désintéressement cultiver un nouvel esprit chevaleresque. Cette politique d'éducation fait nécessité pour Coubertin parce qu'il importe que la nouvelle aristocratie des sociétés démocratiques qui commande le travail apprenne à diriger et se diriger pour qu'elle sorte de la "médiocrité » qui la caractérise, qu'elle prenne les responsabilités de grandeur et de protection qu'implique cette nouvelle liberté. Sans le patronage volontaire, la démocratie marchande laissée "aux économistes et aux calculateurs" favorise le risque de dissociation, la destruction de lien social entre travailleurs et industriels. L'éducation des élites par le sport apparaît comme le remède capable de moraliser la société industrielle 
en produisant un esprit public patriotique nécessaire au maintien de la paix sociale. Suivant la même logique, l'olympisme joue son rôle pédagogique pacificateur à l'échelle internationale: il fait revivre les "mœurs pures» de l'Ancien Régime que le «doux commerce » tend à détruire, en rendant ces heures de gloire aux entreprises héroïques nationales. En cela la pédagogie de Coubertin, anti-utilitaire et élitiste, est mise au service de la puissance nationale : elle entend, au-delà d'une "éthique de la performance", former les élites au désintéressement, à la virilité nationale - la grandeur commerciale et coloniale - à la protection et au contrôle des «faibles", que sont les ouvriers, les indigènes et les femmes.

\section{BIBLIOGRAPHIE}

Aristote (vers 335 avant J. C.) Éthique à Eudème, traduction P. Maréchaux, Paris, Payot et Rivages, 1994.

Aron R. (1962) Dix-huit Leçons sur la société industrielle, Paris, Gallimard.

Aron R. (1967) Les Etapes de la pensée sociologique, Paris, Gallimard.

Aron R. (1960) «Idée politique et vision historique de Tocqueville », Revue française de science politique, vol. $10, \mathrm{n}^{\circ} 3$.

Attali M. \& Chapron T. (2004) « Entre grandeur et désillusion », Illusio, « Jeux olympiques, jeux politiques $» \mathrm{n}^{\circ} 1$, Juin, pp. 61-78.

Audier S. (2004) Tocqueville retrouvé, genèse et enjeux du renouveau tocquevillien français, Paris, Vrin et Editions EHESS,

Ballet J. \& de Bry F (2001) L'Entreprise et l'éthique, Paris, Seuil

Bayle E. \& Mercier S. (2008) « Sport et éthique : enjeux et outils pour le marketing sportif », Cahier du FARGO n ${ }^{\circ} 1080901$

Barbusse B. (2002) «Sport et entreprise : des logiques convergentes ", L'Année sociologique $\mathrm{n}^{\circ} 2$ vol. 52 pp. 391-415

Benoît J. L. (2000) «Anthologie Critique » in A. de Tocqueville, Textes essentiels, Paris, Pocket

Benoît J. L. (2004) Comprendre Tocqueville, Paris, Armand Colin

Bourdieu P. (1980) «Comment peut-on être sportif?», in P. Bourdieu (ed) (1980) Questions de Sociologie, Paris, Éditions de Minuit, pp. 173-195

Boulongne Y. P. (1975) La Vie et l'œuvre pédagogique de Pierre de Coubertin, Ottawa, Editions Leméac Brohm J.M. (1976) Sociologie politique du sport, réédition : Nancy, P.U.N., 1992

Brohm J.M. (1983) Les Jeux Olympiques à Berlin, Bruxelles, Éditions Complexe

Brohm J.M. (2004) «Critique de l'illusion et illusion critique », Illusio, n 1 Juin, « Jeux olympiques, jeux politiques » pp 16-20. 
Brohm J.M. (2008) Pierre de Coubertin, le Seigneur des anneaux. Aux fondements de l'olympisme, Paris, Editions Homnisphères

Burke E. (1790) Réflexions sur le Révolution de France, traduction Pierre Andler, Paris, Hachette, 1989.

Castel R. (1999) Les Métamorphoses de la question sociale : une chronique du salariat, Paris, Gallimard.

Chabot S. (1996) «Éducation civique, instruction publique et liberté de l'enseignement dans l'œuvre d'Alexis de Tocqueville » in L. Guellec (dir) The Tocqueville Review / La Revue Tocqueville Tocqueville et l'esprit de la démocratie, Paris, Les Presses de Sciences Po, 2005, pp. 241-293.

Chanet J. F. (2000) «La fabrique des héros. Pédagogie républicaine et culte des grands hommes de Sedan à Vichy ", Vingtième Siècle. Revue d'histoire, $\mathrm{n}^{\circ}$. 65 Janvier- Mars, pp. 13-34

Chanial P. (2004) « Honneur, vertu et intérêt. Les sociologies de l'association d'Alexis de Tocqueville ", Cahiers internationaux de sociologie $n^{\circ} 2, \mathrm{n}^{\circ} 117$, p. 197-223.

Chapron T. (2004) « Le Sport : un monde fantasmé face aux réalités », in M. Attali (dir.), Le Sport et ses valeurs, Paris, La Dispute, pp. 67-115

Clastres P. (2001) «Pierre de Coubertin et l'Éducation en Angleterre (1888). De la monographie des publics school à la réforme des Lycées Français », Les Études sociales, n 133-1, pp. 47-68

Clastres P. (2003) « La Décennie leplaysienne de Pierre de Coubertin, de la réforme des lycées aux Jeux olympiques (1883-1896) », Les Études sociales, n 137-1 « La Réforme sociale par l'éducation et le sport »

Clastres P. (2004) «La Revue athlétique de Pierre de Coubertin, une revue sportive et libérale, patriotique et coloniale » in F. Carpentier (ed) Le sport est-il éducatif? pp. 34-49, Paris L'Harmattan Clastres P. (2005) «Inventer une élite. Pierre de Coubertin et la chevalerie sportive ", Revue française d'histoire des idées politiques, « Les idées élitistes en 1900 », n²2-2, pp. 275-296

Clastres P. \& Duval N. (2003) « 'Bien armés pour la vie' ou ' Français je suis'. Deux modèles scolaires concurrents : l'École des Roches et le Collège de Normandie » Les Études sociales, n 137-1, pp. 21-33

CNOSF (2006) La raison du plus sport, De la contribution du mouvement sportif à la société française, Septembre.

Coronel de Boissezon J. L. (2007) «La Réinvention du conservatisme politique dans l'histoire des idées politiques » in A. Savoye \& F. Cardoni, Frédéric le Play, parcours, audience et héritage, École des Mines de Paris, pp. 113-156.

Coubertin P. (1887) «L'Education anglaise », Les Études sociales, n 137. 1/2003, pp. 125-144

Coubertin P. (1888) L'Education en Angleterre, Paris, Hachette

Coubertin P. (1913) Essais de psychologie sportive, Grenoble, Éditions Jérôme Million, 1992

Coubertin P. (1920) Pédagogie sportive, Paris, Vrin, 1972

Coubertin P. (1931) Mémoires Olympiques, Paris, Éditions Revue « E.P.S », 1996

Courtney C. P. (1963) Montesquieu and Burke, Oxford, Blackwell,.

Dion M. (1967) « Science sociale et religion chez Frédéric Le Play », Archives des sciences sociales des religions, 24-1, pp. 83-104

Ehrenberg A. (1991) Le Culte de la performance, Paris, Hachette, 
Eyquem M-Th. ( 1966) Pierre de Coubertin, l'épopée olympique, Paris, Calmann-Lévy

Ferry L. (1997) « Olympisme, Humanisme et Démocratie », Actes du Colloque « Congrès du Havre » organisé par le Comité international Pierre de Coubertin.

Gadamer H. G. (1978) L’Idée du Bien comme enjeu platonico-aristotélicien (1978), trad. P. David \& D. Saatdjian, Paris, Vrin, 1994.

Gannett R. (2003) Tocqueville Unveiled, Chicago, University of Chicago Press

Godelier E. (2006) La Culture d'entreprise, Paris, La Découverte.

Guellec L. (1996) Tocqueville et l'apprentissage de la liberté, Paris, Michalon

Haumesser M. (2009) « De quelle matière le concept de sport est-il fait ?» in Moreau \& Taranto, Activité physique et exercices spirituels. Essais de philosophie du sport, Paris, Vrin pp. 47-67

Hirschman A. O. (1977) Les passions et les intérêts. Justifications politiques du capitalisme avant son apogée, Paris, P. U.F. 1980.

d'Iribarne P. (1989) La Logique de l'honneur, gestion des entreprises et traditions nationales, Paris, Seuil d'Iribarne P. (2003) «Trois figures de la liberté » Annales. Histoire, Sciences Sociales, $\mathrm{n}^{\circ} 5$, pp. 953-978

Jamet M. (2002) « Le sport contemporain. Entre l'égalité sociale et l'égalité des chances », Cahiers internationaux de sociologie, $\mathrm{n}^{\circ} 113-2$, pp. 233-260

Jaume L. (2008) Tocqueville. Les sources aristocratiques de la liberté, Paris, Fayard.

Katz-Bénichou G. (2004) « L'éthique sportive est-elle un instrument de marketing ? » Revue française de gestion, $\mathrm{n}^{\circ}$ 150-3, p. 177-192.

Lakoff S. (1998) « Tocqueville, Burke, and the Origins of Liberal Conservatism », Review of Politics, 60-3 pp. 435-464

Le Play F. (1878) La Réforme sociale en France déduite de l'observation comparée des peuples européens, Tours, Alfred Mame et Fils, [sixième édition corrigée et refondue, première édition 1864, trois volumes].

G. Magnane (1964) Sociologie du sport, Paris, Gallimard

Manent P. (2001) Cours familier de philosophie politique, Paris, Gallimard

Montesquieu (1748) L'Esprit des lois, Paris, Garnier Flammarion,1979

Parlebas P. (1986) Éléments de sociologie du sport, Paris, PUF

Putney C. (2003) Muscular Christianity: Manhood and Sports in Protestant America, 1880-1920, Harvard, Harvard University Press

Saint-Martin J. (2004) « La Naissance du sport ou le ramasse mythe des termes modernes (1888-2000)» in M. Attali (dir.), Le Sport et ses valeurs, Paris, La Dispute, pp. 19-65

Salmon A. (2002) Éthique et ordre économique, une entreprise de séduction, Paris, CNRS Editions Salmon A. (2009) Moraliser le capitalisme? Paris, CNRS Éditions

Savoye A. \& Cardoni F. (2007) Frédéric le Play, parcours audience et héritage, École des Mines de Paris Savoye A. (2008) « Frédéric Le Play, concepteur d'une éducation libérale » Le Télémaque, 33, Théories éducatives et réformateurs sociaux, pp. 31-44.

Taine H. (1872) Notes sur l'Angleterre, Paris, Hachette, 1899 
Terret Thierry (2010) Histoire du sport, [2 édition ], Paris, P.U.F.

Tocqueville A. (1835a) De la Démocratie en Amérique I, Paris, Garnier Flammarion, 1992

Tocqueville A. (1835b) Mémoires sur le paupérisme, collection : « Les classiques des sciences

sociales ",

http://classiques.uqac.ca/classiques/De_tocqueville_alexis/memoire_pauperisme_1/

memoire_pauperisme_1.html

Tocqueville A. (1840) De la Démocratie en Amérique II, Paris, Garnier Flammarion, 1992

Tocqueville A. (1858) L'Ancien Régime et la Révolution, Paris, Garnier Flammarion, 1988

Tréanton J-R. (1984) « Faut-il exhumer Le Play ? Ou les héritiers abusifs », Revue française de

sociologie, 25-3, pp. 458-483

Vigarello G. (2002) Du jeu ancien au show sportif, la naissance d'un mythe, Paris, Seuil

Weber E. (1970) «Pierre de Coubertin and the Introduction of Organised Sport in France », Journal of Contemporary History, vol. 5, no. 2, pp. 3-26

Weber E. (1970-1971) « Pierre de Coubertin et les débuts de l'organisation du sport en France », Revue olympique, $\mathrm{n}^{\circ} 34-35$, pp. 355-363 ; n 36, pp. 461-469 ; $\mathrm{n}^{\circ} 40-41$, pp. 64-69 ; $\mathrm{n}^{\circ} 42$, pp. 149-155.

\section{NOTES}

1. Les historiens s'accordent sur le fait que le sport apparaît à la fin du XVIIIe siècle en Angleterre avec la Révolution industrielle et la naissance du capitalisme. À la fin du XIXe siècle, le sport désigne un ensemble de pratiques codifiées et institutionnalisées, sous la forme de clubs locaux, de fédérations nationales et internationales, qui donnent le plus souvent lieu à des compétitions (Terret 2010 pp.3-10). Au-delà de cet accord sur les origines historiques du développement de la pratique du sport, sa définition a fait l'objet d'un débat plus controversé. Apparemment anodin parce que banalement utilisé, le sport a cependant été différemment défini par des historiens comme des sociologues, des psychologues comme des philosophes; si de nombreux points communs se retrouvent dans chacune des définitions, il n'est pas possible de considérer qu'elles offrent une dimension unifiée et arrêtée (Terret 2010 pp. 3-10, Haumesser 2009 pp. 47-67). Aussi afin d'asseoir notre argumentation nous nous appuierons sur la définition que Coubertin propose dans la préface de son traité de Pédagogie sportive (1920) : « Le sport est le culte volontaire et habituel de l'effort musculaire intensif appuyé sur le désir de progrès et pouvant aller jusqu'au risque. Il doit être pratiqué avec ardeur, je dirai même avec violence. Le sport, ce n'est pas l'exercice physique bon pour tous à condition d'être sage et modéré ; le sport est le plaisir des forts, ou de ceux qui veulent le devenir ». Il convient toutefois d'ajouter qu'il manque à cette définition un élément essentiel de la pensée de Coubertin qui s'accorde avec les définitions proposées notamment par Magnane (1964), Brohm (1976) et Parlebas (1981) : à savoir le caractère codifié et la dimension institutionnelle qui confèrent à une activité physique son statut de sport (Terret 2010 p. 8)

2. Le titre complet de l'ouvrage de Le Play, La Réforme sociale en France déduite de l'observation comparée des peuples européens, résume à lui seul la démarche : observer, comparer et juger les faits, pour réformer. Cette méthode "aussi vieille que l'espèce humaine » est explicitement empruntée à Aristote dans la Réforme sociale (Le Play 1887 p. 64). A propos de La Démocratie en Amérique, Aron souligne que le comparatisme de Tocqueville s'accompagne toujours d'un jugement duquel ensuite découle sa conception libérale de la démocratie (Aron 1967 p. 239). Le triptyque observation/biens multiples/bien commun se retrouve également au cœur de la 
critique qu'Aristote adresse à Platon. Dans l'Ethique à Eudème, Aristote récuse l'idée platonicienne selon laquelle il puisse exister un Bien unique et universel. Aussi l'objet de sa démonstration estil de parvenir à définir un bien contingent adapté au réel, multiple. Malgré la tentative de se dégager de la théorie platonicienne, Gadamer relève l'aporie du système : Aristote se reconnaît malgré lui platonicien, en identifiant parmi les biens multiples ce qui pourrait être commun à tous. Il recourt à une généralité dégagée de l'expérience, une Idée a priori du Bien (Ethique à Eudème , I. VII-VIII ; Gadamer 1978 pp. 122-124).

3. Dans «Critique de l'illusion et illusion critique », Jean-Marie Brohm (2004) présente l'objet et la méthode du courant sociologique "Théorie critique du sport» dont il est le chef de file. Critique vis-à-vis de la pratique du sport et de sa pédagogie, il dénonce vivement «l'idéologie sport ", comme un instrument de contrôle social. À propos de Coubertin, il rappelle dans son dernier ouvrage Le Seigneur des anneaux qui lui est consacré (2008) comme dans les Jeux Olympiques à Berlin (1983), que le rénovateur des Jeux n'avait pas renié la version de 1936 orchestrée par l'Allemagne nazie (1983 pp. 156-163, 2008 pp. 111-122).

4. Dans « Les Trois figures de la liberté », d'Iribarne dialogue avec son ouvrage de terrain sur les formes culturelles de management, La Logique de l'honneur (1989). Il confronte trois auteurs à leur culture, Locke, Kant et Tocqueville et distingue trois conceptions de la liberté en tant qu'elles sont porteuses «de conceptions différenciées de l'organisation sociale et politique» et influencent «le cadre institutionnel des rapports de travail»: la liberté anglo-saxonne comme expression du propriétaire (Locke), la liberté allemande comme voix au chapitre dans une communauté (Kant), la liberté française ancrée dans la noblesse (Tocqueville).

\section{INDEX}

Code JEL A12 - Relation of Economists to Other Disciplines, B15 - Historical • Institutional • Evolutionary

\section{AUTEUR}

\section{SHIRINE SABÉRAN}

Chercheur associé, PHARE, Université Paris 1 Panthéon-Sorbonne, Professeur-assistant, Paris ESLSCA Business School 